\title{
Cytokine Gene Polymorphisms and Parkinson's Disease: A Meta-Analysis
}

\author{
Chu Ketan, Zhou Xiao, Luo Ben-yan
}

\begin{abstract}
Background: Cytokines, which are involved in immunological responses, play important role in the development and progression of Parkinson's disease (PD). The functional polymorphisms identified in cytokine genes are thought to influence PD risk. However, the findings of studies investigating the association between cytokine gene polymorphisms and PD risk are still controversial. Therefore, we conducted a meta-analysis, in order to investigate the potential associations between cytokine gene polymorphisms and PD. Methods: Studies of PD and cytokine polymorphisms were identified by searches of PubMed and PDGene. Pooled analyses were performed to assess the association between cytokine gene polymorphisms and PD. Results: Our results indicated a positive association of TNF $\alpha-1031 \mathrm{CC}$ genotype in overall analysis(CC vs. TT: OR=3.146; 95\%CI: 1.631-6.070, p=0.008; CC vs. CT+TT: OR=3.187; 95\%CI: 1.657-6.128, $\mathrm{p}=0.008)$, and an Asian subgroup, $\mathrm{C}$ variant $(\mathrm{OR}=1.328 ; 95 \% \mathrm{CI}: 1.053-1.675$, $\mathrm{p}=0.034)$ also conveyed an increased PD risk as well as CC genotype ( CC vs. TT: OR=3.207; 95\%CI: 1.614-6.373, p=0.004; CC vs. CT+TT: OR=3.238; 95\%CI: $1.636-$ $6.410, \mathrm{p}=0.004)$. A decreased risk for PD was associated with IL-6-174C allele (OR=0.761; 95\%CI: 0.641-0.903, p=0.008) and IL-1RA VNTR 2 allele(OR=0.641; 95\%CI: 0.456-0.826 p=0.004). For the polymorphisms of IL-1 $\beta$ C[-511]T, IL-1 $\alpha$ C[-889]T , TNF $\alpha$ G[308]A, and IL-10 G[-1082]A no significant association was found between the gene polymorphisms and PD risk. Conclusions: Our meta-analysis suggested that gene polymorphisms of TNF $\alpha-1031$, IL-6-174 and IL-1RA VNTR may be associated with PD risk. However, more large well-designed studies will be necessary to validate our findings.
\end{abstract}

RÉSUMÉ: Polymorphismes des gènes des cytokines et maladie de Parkinson : Méta-analyse. Contexte : Les cytokines sont impliquées dans la réponse immunitaire et elles jouent un rôle important dans l'apparition et la progression de la maladie de Parkinson (MP). On pense que les polymorphismes fonctionnels identifiés dans les gènes des cytokines influencent le risque d'être atteint de la MP. Cependant les résultats des études sur ce sujet demeurent controversés. Nous avons donc procédé à une méta-analyse afin d'étudier l'association potentielle entre des polymorphismes des gènes des cytokines et la maladie de Parkinson. Méthode : Nous avons identifié les études sur la MP et les polymorphismes des cytokines au moyen d'une recherche dans PubMed et PDGene. Des analyses des résultats combinés de ces études ont été effectuées pour évaluer l'association entre les polymorphismes des gènes des cytokines et la MP. Résultats : Nos résultats indiquent qu'il existe une association positive du génotype CC de TNF $\alpha$ 1031 à l'analyse globale (CC vs TT : RC = 3,146; IC à 95\% 1,631 à 6,070, p = 0,008; CC vs CT + TT : RC = 3,187; IC à 95\% 1,657 à 6,128, p = $0,008)$ et dans le sous-groupe asiatique, la variante $\mathrm{C}(\mathrm{RC}=1,328$; IC à 95\% 1,053 à $1,675, \mathrm{p}=0,034)$ comportait également un risque plus élevé de MP ainsi que le génotype $\mathrm{CC}(\mathrm{CC}$ vs TT : RC = 3,207; IC à 95\% 1,614 à 6,373; = 0,004; CC vs CT +TT : RC = 3,238; IC à 95\% : 1,636 à 6,410, p $=0,004)$. Un risque plus bas de MP était associé à l'allèle C d'IL-6-174 $(\mathrm{RC}=0,761: \mathrm{IC}$ à $95 \%: 0,641$ à $0,903, \mathrm{p}=0,008)$ et au polymorphisme VNTR du 2e intron d'IL-1RA $(\mathrm{RC}=0,641$; IC à $95 \%: 0,456$ à $0,826, \mathrm{p}=0,004)$. Aucune association significative n'a été constatée entre la MP et les polymorphismes C[-511]T d'IL-1 $\beta$, C[-889]T d'IL-1 $\alpha$, G[-308]A de TNF $\alpha$ et G[-1982]A d'IL-10. Conclusions : Notre méta-analyse suggère que des polymorphismes de TNF $\alpha$-1031, d'Il-6-174 et des VNTR d'IL-1RA pourraient être associés au risque de MP. Cependant nos constatations devront être validées par d'autres grandes études bien conçues.

Can. J. Neurol. Sci. 2012; 39: 58-64

Parkinson Disease (PD) is the second most common neurodegenerative disease and is characterized by a progressive loss of dopaminergic neurons in the substantina nigra region of the brain. The exact cause of the neuronal loss is still largely unknown ${ }^{1}$. For the past decade, accumulating evidence has proved that chronic inflammation of the brain may play a crucial role in neuronal death. Several studies suggested that infection can increase the risk of developing $\mathrm{PD}^{2-4}$. Activated microglia and increased levels of inflammatory mediators have been detected in the striatum of PD patients $\mathrm{s}^{5,6}$ and animal models $\mathrm{s}^{7,8}$. Epidemiological studies also indicated that using nonsteroidal anti-inflammatory drugs (NSAIDs) could reduce the risk of developing $\mathrm{PD}^{9,10}$.

Neuroinflammatory processes might be involved in the progression of neuronal degeneration by producing inflammatory molecules, such as cytokines. Previous studies have shown that the levels of tumor necrosis factor- $\alpha$ (TNF $\alpha$ ), interleukin-1 $\beta$ (IL-1 $\beta$ ) and interleukin-6 (IL-6) were elevated in the serum, cerebrospinal fluid(CSF) and postmortem brain of PD patients $^{1,11-13}$. Several kinds of cytokine receptors are expressed by midbrain dopaminergic neurons ${ }^{14,15}$, and in vitro study of PD

From the Department of Neurology, First Affiliated Hospital, Zhejiang University School of Medicine, Hangzhou, China.

Received June 6, 2011. Final Revisions Submitted August 12, 2011. Correspondence to: Ben-yan Luo, Department of Neurology, First Affiliated Hospital, Zhejiang University School of Medicine, 76\# Qingchun Riad, Hangzhou, 310003, China. 
showed that neuroprotection can be achieved by using neutralizing antibody for these receptors ${ }^{15}$.

In recent years, interests in interactions between inflammatory cytokines and PD have spurred a great number of genetic studies on associations between polymorphisms of cytokine genes and PD. These genes mainly included interleukin- $1 \alpha$ (IL- $1 \alpha)^{16-25}$, IL-1 $\beta^{16,18,20,22,25-27}$, IL-1 receptor antagonist (IL-1RA) ${ }^{16,20,25}, \mathrm{TNF}^{24,27-32}$, IL-6 $^{24,30,33}$, interleukin10 (IL-10) $)^{24,32,34}$ etc. The results of these studies were quite controversial. A previous meta-analysis by Liu et al indicated that polymorphisms of IL- $1 \alpha-889$ and IL- $1 \beta-511$ were not associated with increased PD risk. However, gene effects were evaluated only in a dominant model and recent emerging studies $^{25,35}$ showed a protective effect of IL-1 $\beta$-511 TT genotype. ${ }^{25}$ It is also difficult to evaluate the effects of the other cytokine gene polymorphisms based on separate studies. We therefore conducted a comprehensive meta-analysis to summarize the findings of separate studies exploring the associations between polymorphisms in cytokine genes and PD risk.

\section{Materials ANd Methods}

\section{Selection of studies}

A comprehensive PubMed search up to May 2011 was conducted, using the keywords: "Parkinson", "Parkinson's disease", "interleukin", "IL", "tumor necrosis factor", "TNF", "cytokine" and "polymorphism". We also searched the PDGene for studies investigating IL or TNF gene polymorphisms. The retrieved abstracts were read to identify eligible studies investigating the associations between cytokine polymorphisms and PD risk. Then the full texts were read to assess their appropriateness for inclusion. The references of all

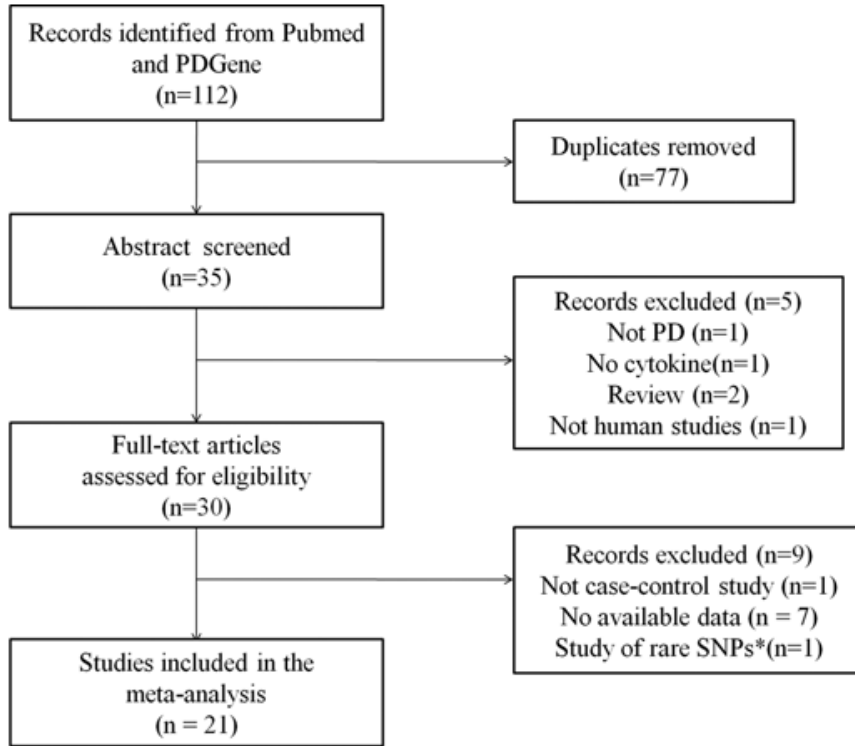

Figure: Flow diagram of the selection of eligible studies. ${ }^{*}$ Rare SNPS are the SNPs studied by less than three studies, and these SNPs were not selected for pooling. identified publications were reviewed for any additional studies not indexed by PubMed and PDGene. Criteria for the inclusion in the analysis were: case-control studies, sufficient data was provided to estimate the association in the form of odds ratio (OR). We contacted authors when there were questions regarding their studies.

\section{Data extraction}

Two reviewers independently extracted the data for analysis. The results were compared and disagreements were resolved by consensus. For overlapping and reused data, only the largest study was included.

\section{Statistical analysis}

For each gene variant, pooled OR along with the $95 \%$ confidence interval $(\mathrm{CI})$ was calculated to measure the strength of the genetic associations with PD. The random effects model was applied if between-studies heterogeneity was present otherwise the fixed effects model was applied. The betweenstudies heterogeneity was assessed with chi-square-based Q test and I-square test ${ }^{36}$. We computed the genetic contrast of the rare allele versus the common type (allele comparison), the associations of the genotype and PD in homozygote comparison, dominant, and recessive model. For IL -1RA Variable Number of Tandem Repeats (VNTR), L signified the long alleles including allele 1,3, 4, and 5, and was considered as the common allele compared to allele $2^{37}$. Stratified analysis based on the racial descent of subjects and Hardy-Weinberg equilibrium (HWE) status (Sensitivity analysis) in control group were also conducted if there were two or more studies included. Publication bias was assessed using the Egger's regression method ${ }^{38}$ for the analysis including more than eight studies. For multiple testing, we used

\section{Table 1: The list and the characteristics of studies that were included in the meta-analysis}

\begin{tabular}{|c|c|c|c|c|c|c|}
\hline Authors & Year & Country & Racial descent & Case & Control & Gene, polymorphism \\
\hline Nishimura et al. & 2000 & Japanese & Asian & 122 & 112 & IL-1 $\alpha$ C[-889]T, IL-1 $\beta$ C[-511]T, IL-1RA VNTR \\
\hline Krüger et al. & 2000 & Germany & Caucasian & 237 & 177 & $\mathrm{TNF} \alpha \mathrm{G}[-308] \mathrm{A}$ \\
\hline Nishimura et al. & 2001 & Japanese & Asian & 172 & 157 & TNFa T[-1031]C \\
\hline Dodel et al. & 2001 & Germany & Caucasian & 201 & 197 & IL-1 $\alpha$ C[-889]T \\
\hline McGeer et al. & 2002 & Canada & Caucasian & 100 & 100 & $\mathrm{IL}-1 \alpha \mathrm{C}[-889] \mathrm{T}, \mathrm{IL}-1 \beta \mathrm{C}[-511] \mathrm{T}$ \\
\hline Schulte et al. & 2002 & Germany & Caucasian & 257 & 260 & IL-1 $\alpha$ C[-889]T, IL-1 $\beta$ C[-511]T \\
\hline Mattila et al. & 2002 & Finland & Caucasian & 52 & 73 & IL-1 $\alpha$ C[-889]T, IL-1 $\beta$ C[-511]T, IL-1RA VNTR \\
\hline Möller et al. & 2004 & Germany & Caucasian & 176 & 170 & IL-1 $\alpha$ C[-889]T \\
\hline Ross et al. & 2004 & Ireland & Caucasian & 90 & 93 & IL-6 C[-174]G, TNF $\alpha$ G[-308]A \\
\hline Håkansson et al. & 2005 & Sweden & Caucasian & 265 & 308 & IL-6 C[-174]G \\
\hline Håkansson et al. & 2005 & Sweden & Caucasian & 265 & 308 & $\mathrm{IL}-10 \mathrm{G}[-1082] \mathrm{A}$ \\
\hline Nishimura et al. & 2005 & Japanese & Asian & 361 & 257 & IL-1 $1 \beta$ C[-511]T \\
\hline Wu et al. & 2007 & Taiwan & Asian & 493 & 388 & $\mathrm{IL}-1 \alpha \mathrm{C}[-889] \mathrm{T}, \mathrm{IL}-1 \beta \mathrm{C}[-511] \mathrm{T}$ \\
\hline Wahner et al. & 2007 & USA & Mixed & 289 & 269 & IL-1 $\beta$ C[-511]T, TNF $\alpha$ G[-308]A \\
\hline Bialecka et al. & 2007 & Poland & Caucasian & 341 & 315 & IL-10 G[-1082]A \\
\hline Wu et al. & 2007 & Taiwan & Asian & 369 & 326 & TNF $\alpha$ G[-308]A, TNF $\alpha[-1031] \mathrm{C}$ \\
\hline Bialecka et al. & 2008 & Poland & Caucasian & 316 & 300 & TNF $\alpha$ G[-308]A, IL-10 G[-1082]A \\
\hline Zhou et al. & 2008 & China & Asian & 533 & 530 & IL-1 $\alpha$ C[-889]T \\
\hline \multirow[t]{4}{*}{ Infante et al. } & 2008 & Spain & Caucasian & 195 & 170 & IL-1 $\alpha$ C[-889]T \\
\hline & & & & 196 & 170 & IL-6 C[-174]G \\
\hline & & & & 193 & 168 & IL-10 G[-1082]A \\
\hline & & & & 194 & 170 & TNFa T[-1031]C \\
\hline Arman et al. & 2010 & Turkey & Other ${ }^{*}$ & 166 & 199 & IL-1 $\alpha$ C[-889]T, IL-1 $\beta$ C[-511]T, IL-1RA VNTR \\
\hline Pascale et al. & 2010 & Italy & Caucasian & 146 & 156 & IL-1 $\beta$ C[-511]T, TNF $\alpha$ G[-308]A, IL-10 G[-1082]A \\
\hline
\end{tabular}

* Other represented for Turkish population in Arman's study ${ }^{25}$ 
the stepdown Bonferroni method to correct the p-values ${ }^{39}$. After correcting, $\mathrm{p}<0.05$ was considered significant. All p-values were two-sided. Data was analyzed using the statistical analysis software stata 11.0 (StataCorp, College Station, TX, USA).

\section{RESULTS}

After a careful screening of the published literature (Figure), twenty-one studies (Table 1) were identified, all of them were published in the English language. Because of the limited number of studies, some of the rare single-nucleotide polymorphisms (SNPs) (studied by less than three studies) investigated in these studies were excluded. Finally, seven gene polymorphisms: IL-1 $\alpha-889$; IL-1 $\beta$-511; IL-1RA VNTR; TNF $\alpha$ -308, -1031; IL-10 -1082 and IL-6 -174 polymorphisms were selected for pooling (Table 2). The extracted data for metaanalysis of the seven gene polymorphisms were shown in Table 2.

\section{IL-1 2 C[-889]T polymorphism (rs1800587)}

Ten case-control studies investigating the association of IL$1 \alpha \mathrm{C}[-889] \mathrm{T}$ polymorphism and PD risk were eligible for our meta-analysis, but one study ${ }^{21}$ was excluded because it used the data overlapping with Dodel's study ${ }^{17}$. In the remaining nine studies, three ${ }^{16,22,23}$ were conducted in Asian populations, five ${ }^{17-}$ 20,24 in Caucasian populations and one ${ }^{25}$ in a Turkish population.

Table 2: Genotype and allele distributions in case and control group

\begin{tabular}{|c|c|c|c|c|c|c|c|c|c|c|c|c|}
\hline \multirow{2}{*}{$\frac{\text { gene polymorphism }}{\text { IL-1 } \alpha \mathrm{C}[-889] \mathrm{T}}$} & \multirow[t]{2}{*}{ Study } & \multirow[t]{2}{*}{ Racial descent } & \multicolumn{5}{|c|}{ Case } & \multicolumn{5}{|c|}{ Control } \\
\hline & & & $\mathrm{C}$ & $\mathrm{T}$ & $\mathrm{CC}$ & $\mathrm{CT}$ & TT & $\mathrm{C}$ & $\mathrm{T}$ & $\mathrm{CC}$ & $\mathrm{CT}$ & TT \\
\hline & Dodel et al. 2001 & Caucasian & 302 & 100 & 108 & 86 & 7 & 307 & 87 & 115 & 77 & 5 \\
\hline & McGeer et al. 2002 & Caucasian & 123 & 77 & 36 & 51 & 13 & 134 & 66 & 37 & 60 & 3 \\
\hline & Schulte et al. 2002 & Caucasian & 357 & 157 & 125 & 107 & 25 & 375 & 145 & 141 & 93 & 26 \\
\hline & Mattila et al. 2002 & Caucasian & 76 & 28 & 28 & 20 & 4 & 95 & 51 & 33 & 29 & 11 \\
\hline & Infante et al. 2008 & Caucasian & 282 & 108 & 102 & 78 & 15 & 255 & 85 & 97 & 61 & 12 \\
\hline & Nishimura et al. 2000 & Asian & 218 & 26 & 97 & 24 & 1 & 199 & 25 & 87 & 25 & 0 \\
\hline & Wu et al. 2007 & Asian & 907 & 79 & 417 & 73 & 3 & 720 & 56 & 334 & 52 & 2 \\
\hline & Zhou et al. 2008 & Asian & 981 & 85 & 451 & 79 & 3 & 947 & 113 & 422 & 103 & 5 \\
\hline & Arman et al. 2009 & Other $^{*}$ & 233 & 95 & 82 & 69 & 13 & 268 & 130 & 94 & 80 & 25 \\
\hline \multirow[t]{9}{*}{ IL-1 $\beta$ C[-511]T } & & & $\mathrm{C}$ & $\mathrm{T}$ & $\mathrm{CC}$ & CT & TT & $\mathrm{C}$ & $\mathrm{T}$ & $\mathrm{CC}$ & $\mathrm{CT}$ & TT \\
\hline & McGeer et al. 2002 & Caucasian & 111 & 89 & 33 & 45 & 22 & 141 & 59 & 44 & 53 & 3 \\
\hline & Schulte et al. 2002 & Caucasian & 361 & 229 & 110 & 141 & 44 & 359 & 175 & 127 & 105 & 35 \\
\hline & Mattila et al. 2002 & Caucasian & 75 & 29 & 25 & 25 & 2 & 78 & 68 & 24 & 30 & 19 \\
\hline & Pascale et al. 2010 & Caucasian & 185 & 107 & 65 & 55 & 26 & 182 & 130 & 56 & 70 & 30 \\
\hline & Nishimura et al. 2005 & Asian & 363 & 359 & 91 & 181 & 89 & 266 & 248 & 69 & 128 & 60 \\
\hline & Wu et al. 2007 & Asian & 549 & 437 & 146 & 257 & 90 & 405 & 371 & 105 & 195 & 88 \\
\hline & Wahner et al. 2007 & Mixed & 347 & 231 & 106 & 135 & 48 & 362 & 176 & 118 & 126 & 25 \\
\hline & Arman et al. 2009 & Other $^{*}$ & 196 & 136 & 55 & 86 & 25 & 206 & 192 & 61 & 84 & 54 \\
\hline \multirow[t]{7}{*}{$\mathrm{TNF} \alpha \mathrm{G}[-308] \mathrm{A}$} & & & G & A & GG & GA & AA & G & A & GG & GA & AA \\
\hline & Krüger et al. 2000 & Caucasian & 385 & 89 & 153 & 79 & 5 & 297 & 57 & 127 & 43 & 7 \\
\hline & Ross et al. 2004 & Caucasian & 140 & 40 & 60 & 20 & 10 & 139 & 47 & 54 & 31 & 8 \\
\hline & Bialecka et al. 2008 & Caucasian & 516 & 116 & 203 & 110 & 3 & 410 & 190 & 121 & 168 & 11 \\
\hline & Pascale et al. 2010 & Caucasian & 255 & 37 & 115 & 25 & 6 & 263 & 49 & 109 & 45 & 2 \\
\hline & Wu et al. 2007 & Asian & 648 & 90 & 282 & 84 & 3 & 585 & 67 & 262 & 61 & 3 \\
\hline & Wahner et al. 2007 & Mixed & 481 & 97 & 205 & 71 & 13 & 464 & 74 & 201 & 62 & 6 \\
\hline \multirow[t]{5}{*}{ IL-10 G[-1082]A } & & & G & A & GG & GA & AA & G & A & GG & GA & AA \\
\hline & Håkansson et al. 2005 & Caucasian & 242 & 276 & 59 & 124 & 76 & 296 & 318 & 76 & 144 & 87 \\
\hline & Bialecka et al. 2007 & Caucasian & 290 & 392 & 61 & 168 & 112 & 279 & 351 & 63 & 153 & 99 \\
\hline & Infante et al. 2008 & Caucasian & 227 & 159 & 66 & 95 & 32 & 191 & 145 & 55 & 81 & 32 \\
\hline & Pascale et al. 2010 & Caucasian & 114 & 178 & 19 & 76 & 51 & 124 & 188 & 27 & 70 & 59 \\
\hline \multirow[t]{4}{*}{ TNF $\alpha$ T[-1031]C } & & & $\mathrm{T}$ & $\mathrm{C}$ & TT & $\mathrm{TC}$ & $\mathrm{CC}$ & $\mathrm{T}$ & $\mathrm{C}$ & TT & TC & $\mathrm{CC}$ \\
\hline & Infante et al. 2008 & Caucasian & 365 & 23 & 174 & 17 & 3 & 316 & 24 & 147 & 22 & 1 \\
\hline & Nishimura et al. 2001 & Asian & 273 & 71 & 112 & 49 & 11 & 274 & 40 & 120 & 34 & 3 \\
\hline & Wu et al. 2007 & Asian & 602 & 136 & 260 & 82 & 27 & 546 & 106 & 228 & 90 & 8 \\
\hline \multirow[t]{4}{*}{ IL-6 G[-174]C } & & & G & $\mathrm{C}$ & GG & $\mathrm{GC}$ & $\mathrm{CC}$ & G & $\mathrm{C}$ & GG & $\mathrm{GC}$ & $\mathrm{CC}$ \\
\hline & Ross et al. 2004 & Caucasian & 96 & 84 & 26 & 44 & 20 & 114 & 72 & 32 & 50 & 11 \\
\hline & Håkansson et al. 2005 & Caucasian & 285 & 231 & 78 & 129 & 51 & 298 & 318 & 68 & 162 & 78 \\
\hline & Pascale et al. 2010 & Caucasian & 257 & 135 & 88 & 81 & 27 & 205 & 135 & 62 & 81 & 27 \\
\hline \multirow[t]{4}{*}{ IL-1RA VNTR } & & & $\mathrm{L}$ & 2 & LL & L2 & 22 & $\mathrm{~L}$ & 2 & LL & $\mathrm{L} 2$ & 22 \\
\hline & Mattila et al. 2002 & Caucasian & 83 & 21 & 31 & 21 & 0 & 96 & 50 & 32 & 32 & 9 \\
\hline & Nishimura et al. 2000 & Asian & 227 & 17 & 105 & 17 & 0 & 216 & 6 & 105 & 6 & 0 \\
\hline & Arman et al. 2009 & Other ${ }^{*}$ & 254 & 74 & 99 & 56 & 9 & 301 & 97 & 120 & 61 & 18 \\
\hline
\end{tabular}

* Other represented for Turkish population in Arman's study ${ }^{25}$ 
A total of 2117 cases and 2029 controls were included. Genotype distribution in the controls of all studies but one ${ }^{18}$ was in agreement with HWE. No evidence for publication bias and heterogeneity was found, so a fixed effects model was adopted for the comparisons.

Only one ${ }^{23}$ of the nine case-control studies found the $\mathrm{T}$ allele was associated with a reduced risk of PD (OR: 0.72, 95\% CI: 0.54-0.97, $\mathrm{p}=0.033)$. In our meta-analysis, no significant association was found in any genetic model. Subgroup analysis of studies enrolling Caucasians and Asians separately as well as sensitive analysis produced insignificant results (Table 3).

\section{IL-1ß C[-511]T polymorphism (rs16944)}

For the association between IL-1 $\beta$ C $[-511] \mathrm{T}$ polymorphisms and PD risk, nine studies were retrieved. One of them ${ }^{16}$ was excluded because of overlapping data with a latter study ${ }^{26}$. The remaining eight studies included 1920 cases and 1709 controls. Among them, four studies ${ }^{18-20,35}$ were conducted in populations of Caucasian, two tw $^{226}$ in Asian populations, one ${ }^{25}$ in a Turkish population and one $^{27}$ recruited individuals of mixed ethnic origin. The genotype frequencies of the control arm in two studies were inconsistent with the $\mathrm{HWE}^{18,25}$. There was statistical evidence of heterogeneity in most of the comparisons except the Asian subgroup (Table 2), so fixed effects model or the random effects model was adopted accordingly.

The results of the studies concerning association between IL$1 \beta C[-511] \mathrm{T}$ polymorphism and PD risk were very controversial. McGeer ${ }^{18}$ and Schulte ${ }^{19}$ found a significant increase of the IL$1 \beta \mathrm{T}$ genotype frequency in $\mathrm{PD}$ cases compared with controls $\left(X^{2}=9.65, p=0.0019^{18} ; X^{2}=4.44, p=0.035^{19}\right)$, Wahner ${ }^{27}$ found that the risk of Parkinson's disease doubled in carriers of the TT genotype $\left(\mathrm{OR}=2.26\right.$; 95\%CI: 1.27-4.02). While Mattila ${ }^{20}$ and $\mathrm{Arman}^{25}$ got the opposite results: allele $\mathrm{C}$ increased the PD risk $\left(\mathrm{OR}=8.8\right.$; 95\%CI: $2.0-39.7^{20}$ and $\mathrm{OR}=1.178$, 95\% CI:0.999$\left.1.388^{25}\right)$. No significant association was found in Pascale's research $^{35}$ and the two Asian studies ${ }^{22,26}$.

Our results did not support an effect of IL-1 $\beta$ C [-511]T on the risk of PD, but the pooled estimate was derived from a set of heterogeneous studies. However, no significant association was found in the subgroup analysis of Caucasian groups and Asian groups. The sensitivity analysis after excluding the studies which deviated from HWE did not alter the pattern of the results. Heterogeneity still existed in the analysis of Caucasian and all HWE studies (Table 3).

Table 3: Results of allelic and genotypic association analysis for the seven cytokine gene polymorphisms

\begin{tabular}{|c|c|c|c|c|c|c|c|c|c|c|c|c|c|c|c|}
\hline \multirow[t]{2}{*}{ Gene polymorphism } & \multirow[t]{2}{*}{ Category } & \multirow[t]{2}{*}{$\begin{array}{c}\text { Number } \\
\text { of } \\
\text { studies }\end{array}$} & \multirow[t]{2}{*}{$\begin{array}{c}\text { Number of } \\
\text { cases/controls }\end{array}$} & \multicolumn{3}{|c|}{ Allelic Comparison } & \multicolumn{3}{|c|}{ Homozygote Comparison } & \multicolumn{3}{|c|}{ Dominant model } & \multicolumn{3}{|c|}{ Recessive model } \\
\hline & & & & $\mathrm{OR}(95 \% \mathrm{CI})$ & $\mathrm{P}^{\prime}$ & $\mathrm{P}^{*}(12 \%)$ & OR $(95 \% \mathrm{CI})$ & $\mathrm{P}^{*}$ & $\mathrm{P}^{*}(\mathrm{I} 2 \%)$ & OR $(95 \% \mathrm{CI})$ & $\mathrm{P}^{e}$ & $\mathrm{P}^{*}(12 \%)$ & $\mathrm{OR}(95 \% \mathrm{CI})$ & $\mathrm{P}^{*}$ & $\mathrm{P}^{*}(\mathrm{I} 2 \%)$ \\
\hline \multirow[t]{5}{*}{$\mathrm{IL}-1 \alpha \mathrm{C}[-889] \mathrm{T}$} & All & 9 & $2117 / 2029$ & $1.000(0.890-1.122)$ & 1 & $0.229(31.1)$ & $1.015(0.736-1.401)$ & 1 & $0.251(21.6)$ & $1.002(0.872-1.152)$ & 1 & $0.302(15.8)$ & $0.985(0.721-1.345)$ & 1 & $0.229(24.1)$ \\
\hline & Caucasian & 5 & $803 / 800$ & $1.118(0.958-1.306)$ & 0.624 & $0.478(0)$ & $1.211(0.821-1.784)$ & 0.668 & $0.162(38.9)$ & $1.153(0.946-1.405)$ & 0.624 & $0.692(0)$ & $1.154(0.793-1.678)$ & 0.668 & $0.119(45.5)$ \\
\hline & Asian & 3 & $1148 / 1030$ & $0.877(0.710-1.082)$ & 0.816 & $0.178(42.1)$ & $0.883(0.318-2.448)$ & 1 & $0.62(0)$ & $0.864(0.619-1.082)$ & 0.816 & $0.183(41.1)$ & $0.909(0.328-2.523)$ & 1 & $0.643(0)$ \\
\hline & Other ${ }^{s}$ & 1 & $166 / 199$ & - & - & - & - & - & - & - & - & - & - & - & - \\
\hline & HWE & 8 & 1929/2017 & $0.979(0.867-1.101)$ & 1 & $0.178(31,3)$ & $0.898(0.640-1.259)$ & 1 & $0.652(0)$ & $1.000(0.866-1.154)$ & 1 & $0.22(26.2)$ & $0.855(0.615-1.188)$ & 1 & $0.775(0)$ \\
\hline \multirow[t]{6}{*}{$\mathrm{IL}-1 \beta \mathrm{C}[-511] \mathrm{T}$} & All & 8 & 1920/1709 & $0.993(0.785-1.256)$ & 1 & $0(82.2)$ & $1.018(0.607-1.706)$ & 1 & $0(82.6)$ & $1.022(0.801-1.304)$ & 1 & $0.006(64.3)$ & $0.997(0.635-1.565)$ & 1 & $0(81.6)$ \\
\hline & Caucasian & 4 & $593 / 596$ & $0.998(0.606-1.645)$ & 1 & $0(87)$ & $1.085(0.337-3.498)$ & 1 & $0(89.7)$ & $1.012(0.603-1.697)$ & 1 & $0.005(76.5)$ & $1.090(0.383-3.104)$ & 1 & $0(85.2)$ \\
\hline & Asian & 2 & $872 / 645$ & $0.908(0.786-1.049)$ & 0.736 & $0.08(67.5)$ & $0.821(0.614-1.098)$ & 0.736 & $0.074(68.7)$ & $0.891(0.710-1.118)$ & 0.736 & $0.169(47.2)$ & $0.864(0.675-1.105)$ & 0.736 & $0.127(56.9)$ \\
\hline & Other ${ }^{8}$ & 1 & $166 / 199$ & - & - & - & - & - & - & - & - & - & - & - & - \\
\hline & Mixed & 1 & $369 / 389$ & - & - & - & - & - & - & - & - & - & - & - & - \\
\hline & HWE & 6 & $1654 / 1410$ & $0.956(0.746-1.224)$ & 1 & $0(80.7)$ & $0.933(0.506-1.538)$ & 1 & $0(78.7)$ & $0.985(0.735-1.319)$ & 1 & $0.004(70.6)$ & $0.959(0.644-1.428)$ & 1 & $0.002(73.1)$ \\
\hline \multirow[t]{5}{*}{ TNF $\alpha$ G[-308]A } & All & 6 & $1447 / 1321$ & $1.105(0.954-1.279)$ & 0.549 & $0.471(0)$ & $1.539(0.958-2.471)$ & 0.296 & $0.323(14.2)$ & $1.072(0.908-1.267)$ & 0.824 & $0.115(43.5)$ & $1.570(0.983-2.509)$ & 0.295 & $0.244(25.4)$ \\
\hline & Caucasian & 4 & $789 / 726$ & $1.026(0.850-1.238)$ & 1 & $0.387(0.9)$ & $1.401(0.821-2.603)$ & 0.594 & $0.175(39.5)$ & $0.970(0.781-1.206)$ & 1 & $0.076(56.3)$ & $1.532(0.867-2.707)$ & 0.508 & $0.118(48.9)$ \\
\hline & Asian & 1 & $369 / 326$ & & - & - & - & & - & - & - & - & - & - & - \\
\hline & Mixed & 1 & $289 / 269$ & - & - & - & - & - & - & - & - & - & - & - & - \\
\hline & HWE & 5 & $1131 / 1021$ & $1.102(0.931-1.305)$ & 1 & $0.335(12.3)$ & $1.309(0.781-2.195)$ & 1 & $0.431(0)$ & $1.087(0.898-1.318)$ & 1 & $0.067(54.3)$ & $1.340(0.804-2.234)$ & 1 & $0.319(14.9)$ \\
\hline $\mathrm{IL}-10 \mathrm{G}[-1082] \mathrm{A}$ & All & 4 & $936 / 949$ & $1.024(0.900-1.165)$ & 1 & $0.836(0)$ & $1.030(0.793-1.337)$ & 1 & $0.715(0)$ & $1.021(0.821-1.271)$ & 1 & $0.868(0)$ & $1.040(0.850-1.271)$ & 1 & $0.868(0)$ \\
\hline \multirow[t]{3}{*}{ TNF $\alpha$ T[-1031]C } & All & 3 & $735 / 653$ & $1.247(1.005-1.547)$ & 0.264 & $0.091(58.4)$ & $3.146(1.631-6.070)$ & 0.008 & $0.919(0)$ & $1.091(0.852-1.397)$ & 0.824 & $0.065(63.5)$ & $3.187(1.657-6.128)$ & 0.008 & $0.977(0)$ \\
\hline & Caucasian & 1 & $194 / 170$ & - & - & - & - & - & - & - & - & - & - & - & - \\
\hline & Asian & 2 & $541 / 483$ & $1.328(1.053-1.675)$ & 0.034 & $0.099(63.2)$ & $3.207(1.614-6.373)$ & 0.004 & $0.717(0)$ & $1.170(0.894-1.531)$ & 0.252 & $0.052(73.4)$ & $3.238(1.636-6.410)$ & 0.004 & $0.886(0)$ \\
\hline IL-6 G[-174]C & All & 3 & $544 / 571$ & $0.761(0.641-0.903)$ & 0.008 & $0.234(31.1)$ & $0.883(0.438-1.777)$ & 0.896 & $0.031(71.3)$ & $0.755(0.585-0.975)$ & 0.093 & $0.175(42.7)$ & $0.890(0.659-1.202)$ & 0.896 & $0.062(64.1)$ \\
\hline \multirow[t]{3}{*}{ IL-1RA VNTR } & $\mathrm{All}^{\mathrm{k}}$ & 2 & $237 / 251$ & $0.614(0.456-0.826)$ & 0.004 & $0.361(0)$ & $0.300(0.137-0.653)$ & 0.006 & $0.168(47.3)$ & $0.637(0.442-0.917)$ & 0.015 & $0.558(0)$ & $0.334(0.155-0.720)$ & 0.01 & $0.180(44.3)$ \\
\hline & Caucasian & 1 & $71 / 52$ & - & - & - & - & - & - & - & - & - & - & - & - \\
\hline & Other ${ }^{5}$ & 1 & $166 / 199$ & - & - & & - & - & - & & & - & - & - & - \\
\hline
\end{tabular}

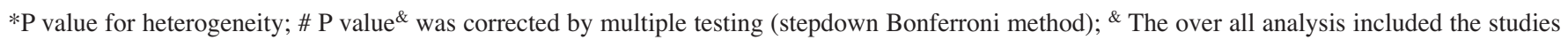
of Mattila's ${ }^{20}$ and Arman's ${ }^{25}$; §Other represented for Turkish population in Arman's study ${ }^{25}$ 


\section{TNF $\alpha$ G[-308]A polymorphism (rs1800629)}

Six studies evaluating the role of the TNFa G[-308]A polymorphism for PD risk were eligible for meta-analysis. One study $^{31}$ was conducted in an Asian population, four ${ }^{28,30,32,35}$ in Caucasian populations and one ${ }^{27}$ recruited individuals of mixed ethnic origin. In total, 1447 cases and 1321 controls were included. Genotype distribution in one study ${ }^{32}$ was not in agreement with HWE. No heterogeneity was found in any of the comparisons, so fixed effects model was adopted.

Even though one study found that GA phenotype was more frequent in patients ${ }^{28}$, our meta-analysis did not find any significant association in all the comparisons (Table 3 ).

\section{IL-10 G[-1082]A polymorphism (rs 1800896)}

There were five case-control studies ${ }^{24,32,34,35,40}$ investigating the association of IL-10 G[-1082]A polymorphism and PD risk. $\mathrm{One}^{32}$ was excluded because of overlapping data ${ }^{40}$. The remaining four studies included 936 PD cases and 949 healthy controls, all of which were Caucasian studies. Heterogeneity was absent in all the comparisons, so we adopted the fixed effects model.

In agreement with the three published studies. Our metaanalysis did not find any significant association between this gene polymorphism and PD risk (Table 3).

\section{TNF $\alpha$ T[-1031]C polymorphism (rs1799964)}

Three studies ${ }^{24,29,31}$ provided data on the association between TNF $\alpha$ T[-1031]C polymorphism and PD, two ${ }^{29,31}$ of them enrolled Asian subjects, and one ${ }^{24}$ was performed in a Caucasian population. Seven hundred and thirty-five cases and 653 controls were included. Genotype distributions in the controls of all studies were in agreement with HWE. No heterogeneity existed in the any of the comparisons, thus a fixed effects model was applied.

Two Asian studies ${ }^{29,31}$ found significant associations between TNF $\alpha$ T[-1031]C polymorphism and PD, while the Caucasian study $^{24}$ did not. In our meta-analysis, the overall OR of the C allele for PD was 1.247 (95\% CI: 1.005-1.547), however, the pvalue of this comparison did not survive multiple test $(\mathrm{P}=0.264$ after correction), both homozygote comparison ( $\mathrm{OR}=3.146,95 \%$ CI: 1.631-6.070, $\mathrm{P}=0.008)$ and recessive model $(\mathrm{OR}=3.187$, 95\% CI:1.657-6.128, $\mathrm{P}=0.008$ ) provided evidence of an association between this SNP and PD. A slightly higher and significant risk for $\mathrm{PD}$ was observed in the Asian subgroups $(\mathrm{C}$ versus $\mathrm{T}: \mathrm{OR}=1.32895 \% \mathrm{CI}: 1.053-1.675, \mathrm{P}=0.034 ; \mathrm{CC}$ versus TT OR=3.207, 95\% CI: $1.614-6.373, \mathrm{P}=0.004$; $\mathrm{CC}$ versus TT+TC: $\mathrm{OR}=3.238,95 \% \mathrm{CI}: 1.636-6.410, \mathrm{P}=0.004)$.

\section{IL-6 G[-174]C polymorphism (rs1800795)}

Three studies $24,30,33$ focused on the potential association between the IL-6 G[-174]C polymorphism and PD. All of them were conducted in Caucasian populations, and a total of 544 cases and 571 controls were included. There was no deviation from HWE. Heterogeneity was considered only in the homozygote comparison, so the random effects model was applied in this comparison.

Only one study ${ }^{33}$ found a significantly elevated frequency of the GG genotype in the patient group $(\mathrm{P}=0.03)$, while the other two got insignificant results. Our meta-analysis indicated that the $\mathrm{C}$ allele has a protective effect $(\mathrm{OR}=0.761,95 \% \mathrm{CI}$ : 0.641 0.903, $\mathrm{p}=0.008)$. The OR in the Dominant model was 1.247 (95\% CI: 1.005-1.547), however the $\mathrm{p}$-value $(\mathrm{p}=0.093)$ of this comparison was not significant after correction.

\section{IL-1RA VNTR}

The effect of this polymorphism on the occurrence of PD has been investigated in three studies, among them was one Asian study $^{16}$, one Caucasian study ${ }^{20}$ and one ${ }^{25}$ Turkish study. In total, 359 PD cases and 363 healthy controls were included. Since the allele 2 frequency was very low (4.9\%), and genotype 22 was not detected in the Asian study, this study was excluded from the overall analysis (Table 3 ). No between-study heterogeneity was detected, thus the fixed effects model was adopted.

In our meta-analysis all of the four comparisons provided evidence for a protective role of allele 2 for $\mathrm{PD}$ without heterogeneity $(\mathrm{OR}=0.614,95 \% \mathrm{CI}: 0.456-0.826, \mathrm{p}=0.004)$ (Table 3).

\section{Discussion}

The current meta-analysis is a comprehensive quantitative evaluation of the relevance to PD of seven cytokine gene polymorphisms. Findings of the meta-analysis indicated a positive association of $\mathrm{TNF} \alpha-1031 \mathrm{CC}$ genotype in overall analysis, and in the Asian subgroup; $\mathrm{C}$ variant, as well as $\mathrm{CC}$ genotype, conveyed an increased PD risk. Several arguments reinforce these findings. TNF $\alpha-1031 \mathrm{C}$ variant was reported to be associated with elevation of TNF $\alpha$ gene transcription ${ }^{41,42}$. Several inflammation-related diseases have been reported to be associated with this SNP, such as Behçet disease and Graves' disease etc. ${ }^{43,44}$. However TNF $\alpha-1031 \mathrm{C}$ is in strong linkage disequilibrium with the $\mathrm{TNF} \alpha-863 \mathrm{~A}$ and $\mathrm{TNF} \alpha$ microsatellite (TNFo-2) allele 2, a high producer of $\mathrm{TNF}^{29}$. We could not exclude the possibility that the positive finding was partly attributed to the linkage between these SNPs. Further studies evaluating the combined effect of two or more SNPs by haplotype analysis are needed to detect the real association between this gene polymorphism and PD.

The current report also documented a decreased risk for PD in IL-6-174C carriers. IL-6 has a pro-inflammatory effect. Transgenic mice over expressing IL-6 developed an age-related neurodegeneration ${ }^{45}$, the methamphetamine-induced neurotoxicity was attenuated in IL-6 knockout mice ${ }^{46}$. Increased levels of IL-6 were found in the CSF and the diseased brain tissue of PD patients ${ }^{1,13}$. IL-6 (-174) C allele has been associated with decreased IL-6 expression ${ }^{24}$. All these might be explanations for our results. However, studies with larger sample sizes are needed to further confirm the gene effect of IL-6 (-174) polymorphism in PD.

In the last section, our study showed a protective role of IL1RA VNTR 2 allele. IL-1RA is an anti-inflammatory cytokine. Previous studies reported that the 2 allele was associated with increased IL-1RA production in human monocytes ${ }^{47}$. However, different allele frequencies of this polymorphism were observed in different ethnic populations. According to our analysis and previous studies $^{48,49}$, the effect of IL-1RA VNTR polymorphism may not be the same between Caucasian and Asian populations. 
Further studies, including subjects of different ethnic background, should be conducted to illustrate the gene effect in PD.

In the present meta-analysis, many efforts have been made to avoid possible sources of bias. We assessed the impact of deviations from the $\mathrm{HWE}^{50}$. Sensitivity analysis that excluded those studies inconsistent with the HWE did not change the pattern of the findings. We performed appropriate tests for detecting publication bias using Egger's test ${ }^{38}$. No significant publication bias was found in any of the analyses. While ethnicity can strongly influence the distribution and effect of gene polymorphisms, we did subgroup analysis according to ethnic background. To investigate the effect of these gene polymorphisms, we not only tested the allele prevalence of each gene, but also performed the analysis in three commonly used genetic models. Finally, we performed multiple testing to control for false discovery rate ${ }^{39}$.

However, several limitations in our meta-analysis should be addressed. First, due to sparse data on age at onset of the disease and sex across studies, we could not investigate the effect of these factors in the gene-disease interaction. Second, certain alleles of some SNPs are in linkage disequilibrium with each other, such as IL-10 G[-1082]A, IL-10 T[-819]C and IL-10 C[592] $\mathrm{A}^{51}$ and $\mathrm{TNF} \alpha-1031$ polymorphism mentioned above. Only two studies ${ }^{32,40}$ examined the composite effect of three IL-10 SNP sites. Therefore, future studies should carefully address such interactions, i.e. perform stratified analyses that could be used in a subsequent meta-analysis and evaluate the combined effect of two or more SNPs, perhaps by haplotype analysis.

In conclusion, we found that TNF $\alpha-1031$, IL-6-174 and IL1RA VNTR polymorphisms are associated with the probability of PD. However, additional large well-designed studies will be necessary to validate our findings.

\section{REFERENCES}

1. Hirsch EC, Hunot S. Neuroinflammation in Parkinson's disease: a target for neuroprotection? Lancet Neurol. 2009; 8(4): 382-97.

2. Ogata A, Tashiro K, Pradhan S. Parkinsonism due to predominant involvement of substantia nigra in Japanese encephalitis. Neurology. 2000; 55(4): 602

3. Ling ZD, Chang Q, Lipton JW, et al. Combined toxicity of prenatal bacterial endotoxin exposure and postnatal 6-hydroxydopamine in the adult rat midbrain. Neuroscience. 2004; 124(3): 619-28.

4. Long-Smith CM, Sullivan AM, Nolan YM. The influence of microglia on the pathogenesis of Parkinson's disease. Prog Neurobiol. 2009; 89(3): 277-87.

5. Imamura K, Hishikawa N, Sawada M, et al. Distribution of major histocompatibility complex class II-positive microglia and cytokine profile of Parkinson's disease brains. Acta Neuropathol. 2003; 106(6): 518-26.

6. Mirza B, Hadberg H, Thomsen P, et al. The absence of reactive astrocytosis is indicative of a unique inflammatory process in Parkinson's disease. Neuroscience. 2000; 95(2): 425-32.

7. Ghosh A, Roy A, Liu X, et al. Selective inhibition of NF-kappaB activation prevents dopaminergic neuronal loss in a mouse model of Parkinson's disease. Proc Natl Acad Sci USA. 2007; 104(47): 18754-9.

8. Wang T, Zhang W, Pei Z, et al. Reactive microgliosis participates in $\mathrm{MPP}+$-induced dopaminergic neurodegeneration: role of $67 \mathrm{kDa}$ laminin receptor. FASEB J. 2006; 20(7): 906-15.

9. Chen H, Zhang SM, Hernan MA, et al. Nonsteroidal antiinflammatory drugs and the risk of Parkinson disease. Arch Neurol. 2003; 60(8): 1059-64.
10. Chen H, Jacobs E, Schwarzschild MA, et al. Nonsteroidal antiinflammatory drug use and the risk for Parkinson's disease. Ann Neurol. 2005; 58(6): 963-7.

11. Blum-Degen D, Muller T, Kuhn W, et al. Interleukin-1 beta and interleukin-6 are elevated in the cerebrospinal fluid of Alzheimer's and de novo Parkinson's disease patients. Neurosci Lett. 1995; 202(1-2): 17-20.

12. Mogi M, Harada M, Riederer P, et al. Tumor necrosis factor-alpha (TNF-alpha) increases both in the brain and in the cerebrospinal fluid from parkinsonian patients. Neurosci Lett. 1994; 165(1-2): 208-10.

13. Mogi M, Harada M, Kondo T, et al. Interleukin-1 beta, interleukin6 , epidermal growth factor and transforming growth factor-alpha are elevated in the brain from parkinsonian patients. Neurosci Lett. 1994; 180(2): 147-50.

14. Mogi M, Togari A, Kondo T, et al. Caspase activities and tumor necrosis factor receptor R1 (p55) level are elevated in the substantia nigra from parkinsonian brain. J Neural Transm. 2000; 107(3): 335-41.

15. Gayle DA, Ling Z, Tong C, et al. Lipopolysaccharide (LPS)induced dopamine cell loss in culture: roles of tumor necrosis factor-alpha, interleukin-1beta, and nitric oxide. Brain Res Dev Brain Res. 2002; 133(1): 27-35.

16. Nishimura M, Mizuta I, Mizuta E, et al. Influence of interleukin1 beta gene polymorphisms on age-at-onset of sporadic Parkinson's disease. Neurosci Lett. 2000; 284(1-2): 73-6.

17. Dodel RC, Lohmuller F, Du Y, et al. A polymorphism in the intronic region of the IL-1alpha gene and the risk for Parkinson's disease. Neurology. 2001; 56(7): 982-3.

18. McGeer PL, Yasojima K, McGeer EG. Association of interleukin-1 beta polymorphisms with idiopathic Parkinson's disease. Neurosci Lett. 2002; 326(1): 67-9.

19. Schulte T, Schols L, Muller T, et al. Polymorphisms in the interleukin-1 alpha and beta genes and the risk for Parkinson's disease. Neurosci Lett. 2002; 326(1): 70-2.

20. Mattila KM, Rinne JO, Lehtimaki T, et al. Association of an interleukin 1B gene polymorphism (-511) with Parkinson's disease in Finnish patients. J Med Genet. 2002; 39(6): 400-2.

21. Moller JC, Depboylu C, Kolsch H, et al. Lack of association between the interleukin-1 alpha (-889) polymorphism and earlyonset Parkinson's disease. Neurosci Lett. 2004; 359(3): 195-7.

22. $\mathrm{Wu} \mathrm{YR}$, Chen $\mathrm{CM}$, Hwang JC, et al. Interleukin-1 alpha polymorphism has influence on late-onset sporadic Parkinson's disease in Taiwan. J Neural Transm. 2007; 114(9): 1173-7.

23. Zhou YT, Yang JF, Zhang YL, et al. Protective role of interlekin-1 alpha gene polymorphism in Chinese Han population with sporadic Parkinson's disease. Neurosci Lett. 2008; 445(1): 23-5.

24. Infante J, Garcia-Gorostiaga I, Sanchez-Juan P, et al. Inflammationrelated genes and the risk of Parkinson's disease: a multilocus approach. Eur J Neurol. 2008; 15(4): 431-3.

25. Arman A, Isik N, Coker A, et al. Association between sporadic Parkinson disease and interleukin-1beta -511 gene polymorphisms in the Turkish population. Eur Cytokine Netw. 21(2): 116-121.

26. Nishimura M, Kuno S, Kaji R, et al. Glutathione-S-transferase-1 and interleukin-1beta gene polymorphisms in Japanese patients with Parkinson's disease. Mov Disord. 2005; 20(7): 901-2.

27. Wahner AD, Sinsheimer JS, Bronstein JM, et al. Inflammatory cytokine gene polymorphisms and increased risk of Parkinson disease. Arch Neurol. 2007; 64(6): 836-40.

28. Kruger R, Hardt C, Tschentscher F, et al. Genetic analysis of immunomodulating factors in sporadic Parkinson's disease. J Neural Transm. 2000; 107(5): 553-62.

29. Nishimura M, Mizuta I, Mizuta E, et al. Tumor ncrosis factor gene polymorphisms in patients with sporadic Parkinson's disease. Neurosci Lett. 2001; 311(1): 1-4.

30. Ross OA, O'Neill C, Rea IM, et al. Functional promoter region polymorphism of the proinflammatory chemokine IL-8 gene associates with Parkinson's disease in the Irish. Hum Immunol. 2004; 65(4): 340-6. 
31. Wu YR, Feng IH, Lyu RK, et al. Tumor necrosis factor-alpha promoter polymorphism is associated with the risk of Parkinson's disease. Am J Med Genet B Neuropsychiatr Genet. 2007; 144B(3): 300-4.

32. Bialecka M, Klodowska-Duda G, Kurzawski M, et al. Interleukin10 (IL10) and tumor necrosis factor alpha (TNF) gene polymorphisms in Parkinson's disease patients. Parkinsonism Relat Disord. 2008; 14(8): 636-40.

33. Hakansson A, Westberg L, Nilsson S, et al. Interaction of polymorphisms in the genes encoding interleukin- 6 and estrogen receptor beta on the susceptibility to Parkinson's disease. Am J Med Genet B Neuropsychiatr Genet. 2005; 133B(1): 88-92.

34. Hakansson A, Westberg L, Nilsson S, et al. Investigation of genes coding for inflammatory components in Parkinson's disease. Mov Disord. 2005; 20(5): 569-73.

35. Pascale E, Passarelli E, Purcaro C, et al. Lack of association between IL-1beta, TNF-alpha, and IL-10 gene polymorphisms and sporadic Parkinson's disease in an Italian cohort. Acta Neurol Scand. 2011; 124(3): 176-81

36. Lau J, Ioannidis JP, Schmid CH. Quantitative synthesis in systematic reviews. Ann Intern Med. 1997; 127(9): 820-6.

37. Xue H, Lin B, Ni P, et al. Interleukin-1B and interleukin-1 RN polymorphisms and gastric carcinoma risk: a meta-analysis. J Gastroenterol Hepatol. 25(10): 1604-17.

38. Egger M, Davey Smith G, Schneider M, et al. Bias in meta-analysis detected by a simple, graphical test. BMJ. 1997; 315(7109): 629-34.

39. Holm S. A simple sequentially rejective multiple test procedure. Scand J Statist. 1979; 6: 65-70.

40. Bialecka M, Klodowska-Duda G, Kurzawski M, et al. Interleukin10 gene polymorphism in Parkinson's disease patients. Arch Med Res. 2007; 38(8): 858-63.

41. Park YJ, Park H, Park MH. TNF-alpha promoter polymorphisms and extended HLA and TNF-alpha haplotypes in Koreans based on 100 families. Tissue Antigens. 2004; 63(1): 75-9.

42. Higuchi T, Seki N, Kamizono S, et al. Polymorphism of the 5'flanking region of the human tumor necrosis factor (TNF)-alpha gene in Japanese. Tissue Antigens. 1998; 51(6): 605-12.
43. Li N, Zhou Z, Liu X, et al. Association of tumour necrosis factor alpha (TNF-alpha) polymorphisms with Graves' disease: A meta-analysis. Clin Biochem. 2008; 41(10-11): 881-6.

44. Touma Z, Farra C, Hamdan A, et al. TNF polymorphisms in patients with Behcet disease: a meta-analysis. Arch Med Res. 41(2):142-6.

45. Heyser CJ, Masliah E, Samimi A, et al. Progressive decline in avoidance learning paralleled by inflammatory neurodegeneration in transgenic mice expressing interleukin 6 in the brain. Proc Natl Acad Sci USA. 1997; 94(4): 1500-5.

46. Ladenheim B, Krasnova IN, Deng X, et al. Methamphetamineinduced neurotoxicity is attenuated in transgenic mice with a null mutation for interleukin-6. Mol Pharmacol. 2000; 58(6): 1247-56.

47. Danis VA, Millington M, Hyland VJ, et al. Cytokine production by normal human monocytes: inter-subject variation and relationship to an IL-1 receptor antagonist (IL-1Ra) gene polymorphism. Clin Exp Immunol. 1995; 99(2): 303-10.

48. Serdaroglu G, Alpman A, Tosun A, et al. Febrile seizures: interleukin 1beta and interleukin-1 receptor antagonist polymorphisms. Pediatr Neurol. 2009; 40(2): 113-6.

49. Chou IC, Lin WD, Wang CH, et al. Interleukin (IL)-1beta, IL-1 receptor antagonist, IL-6, IL-8, IL-10, and tumor necrosis factor alpha gene polymorphisms in patients with febrile seizures. J Clin Lab Anal. 24(3): 154-9.

50. Trikalinos TA, Salanti G, Khoury MJ, et al. Impact of violations and deviations in Hardy-Weinberg equilibrium on postulated gene-disease associations. Am J Epidemiol. 2006; 163(4): 300-9.

51. Thakkinstian A, Dmitrienko S, Gerbase-Delima M, et al. Association between cytokine gene polymorphisms and outcomes in renal transplantation: a meta-analysis of individual patient data. Nephrol Dial Transplant. 2008; 23(9): 3017-23. 